\title{
Markt oder Staat? Kooperationsprobleme in der Europäischen Union $^{1}$
}

Dass es in der Europäischen Union Kooperationsprobleme gibt, ist hinlänglich bekannt. Kooperationsprobleme gibt es bei der Gewährung von Asyl und bei der Regulierung von Banken, die Mitgliedsländer können sich nicht auf die Schaffung einer gemeinsamen, europäischen Flugsicherung und auch nicht auf einheitliche Unternehmenssteuern einigen, um so das Problem der kreativen Steuervermeidung, der legalen Steuerflucht zu lösen. Ich will im Folgenden an die Frage des Kooperationsproblems der EU aus einer allgemeineren, gesellschaftstheoretischen Perspektive herangehen, werde aber auch ein paar praktische Beispiele bringen.

Bei den Kooperationsproblemen der Europäischen Union handelt es sich nicht um eigentlich vermeidbare Mängel: Kooperationsprobleme sind vielmehr der Konstruktion der EU inhärent. Rainer Lepsius hat kürzlich den besonderen Charakter der EU als "paktierte Kooperation ohne Hegemonialmacht « beschrieben. ${ }^{2}$ In der EU müssen eigenständige Staaten miteinander kooperieren. Kooperation ist anspruchsvoller als bloße Koordination, gegenseitige Abstimmung: Kooperieren heißt, bewusst mit anderen auf einen gemeinsamen Zweck hin zusammenzuwirken. Die Existenz eines gemeinsamen Ziels ist die zentrale Voraussetzung von Kooperation. ${ }^{3}$ Das Ziel kann die Lösung eines Problems, die Erledigung einer Aufgabe oder die Maximierung des gemeinsamen Nutzens (public welfare) sein, wichtig ist nur, dass es das gemeinsame Ziel von zwei oder mehr Akteuren ist. Schon die Fähigkeit, ein gemeinsames Ziel überhaupt zu formulieren, setzt ein Wir-Bewusstsein voraus. In der EU ist dieses Bewusstsein prekär, aber es ist keine Frage, dass die EU gemeinsame Ziele hat.

Die EU entstand bekanntlich aus der EG und diese aus der EWG, die ihrerseits auf der Montanunion fußte. Ihr ursprüngliches Ziel war die Schaffung eines gemeinsamen Marktes. Damit wurde auf regionaler Ebene der sogenannte »Washington Consensus « umgesetzt, der globales wirtschaftliches Wachstum durch die Abschaffung von Handelsbeschränkungen und von nationalen Regelungen anstrebte, die die Dynamik der Ökonomie begrenzen. Wer den gemeinsamen europäischen Markt ursprünglich wollte, die Vertreter der Politik oder die Vertreter der Wirtschaft, ist eine Frage für Historiker. Offenbar hatten beide gute Gründe, aber auch Bedenken.

1 Öffentlicher Vortrag, gehalten am 6. November 2013 in der Berlin-Brandenburgischen Akademie der Wissenschaften im Rahmen der Reihe »Europa in der Krise".

2 Lepsius 2013, S. 189.

3 Vgl. den Jahresbericht des Zentrums für globale Kooperationsforschung (Centre for Global Cooperation Research 2013, S. 12-15). 
Für große exportorientierte Unternehmen ging es um Markterweiterung, um die Überschreitung von Grenzen zwischen europäischen Ländern - aber zugleich gab es Widerstand bisher geschützter Branchen vor auswärtiger Konkurrenz. Die Vertreter der Politik hatten eigene Gründe. Sie erhofften sich vom gemeinsamen Markt wirtschaftliches Wachstum und reagierten damit auch auf den zunehmend spürbaren Wettbewerbsdruck aus den USA und Japan. Zugleich gab es vonseiten der Regierungen Widerstand gegen die Abgabe nationaler Regelungskompetenzen und damit den Verzicht auf protektionistische Maßnahmen im Interesse der eigenen Industrie.

Diese anfängliche Ambivalenz erschwerte die Schaffung eines gemeinsamen Marktes. Das ehrgeizige Ziel der Harmonisierung durch eine für alle Mitglieder verbindliche europäische Gesetzgebung ließ sich nur begrenzt durchsetzen. In vielen Bereichen ging es deshalb zunächst nur um die Nicht-Diskriminierung europäischer Konkurrenten, dann etwas anspruchsvoller um gegenseitige Nicht-Behinderung, aus der auf dem Weg über die Entwicklung gemeinsamer Mindeststandards schließlich die heute dominierende wechselseitige Anerkennung nationaler Normen wurde, durch die Marktschranken auch ohne gesetzliche Harmonisierung abgebaut werden. ${ }^{4}$ Mit der Transformation der EWG in die EU hatte sich die ursprüngliche Zielsetzung erweitert; es ging jetzt nicht mehr nur um ökonomische, sondern auch um vorsichtige Schritte in Richtung auf politische Integration. Die EU sollte nicht nur ein gemeinsamer Markt, sondern auch ein politischer Akteur werden, nach innen wie nach außen. ${ }^{5}$ Nach außen verlangt das, in internationalen Gremien und Verhandlungen ein gemeinsames EU-Interesse zu vertreten; ein solches gemeinsames Interesse zu bilden erwies und erweist sich, wie wir wissen, vor allem in Fragen der Außenpolitik als außerordentlich schwierig. Nach innen wurde die EU schrittweise zu einem » Regulierungsstaat $« .{ }^{6}$ Neben politische Interventionen zum Zweck der Marktschaffung traten zunehmend Versuche, durch Regulierung Fehlentwicklungen des gemeinsamen Marktes zu verhindern.

Der Übergang von der Marktschaffung zur Marktregelung fand nur langsam und zögernd statt. ${ }^{7} \mathrm{Ob}$ nicht nur marktschaffend, sondern Marktakteure und ihr Verhalten regelnd interveniert werden soll, war immer wieder eine Streitfrage in der EU. Das gemeinsame Ziel aller EU-Mitglieder, einen integrierten europäischen Wirtschaftsraum zu schaffen, ist unbestimmt und muss, um Entscheidungen leiten zu können, spezifiziert werden: Wie frei soll dieser europäische Markt sein, wie weit und wie soll er im Einzelnen politisch geregelt werden? Bei vielen europapolitischen Kontroversen bildeten sich dabei zwei Koalitionen heraus; trotz wechselnder Grenzen gehörte zu der Koalition, die vor allem Märkte schaffen wollte, meist Großbritannien, während Deutschland meist zu der anderen Koalition gehörte, die Märkte

4 Schmidt 2007.

5 Kratochvil 2013.

6 Vgl. zum Beispiel Eberlein, Grande 2003.

7 Vgl. Scharpf 1999.

Leviathan, 42. Jg., 2/2014 
politisch regeln wollte. ${ }^{8}$ In diesen Debatten stand - und steht - das Prinzip » Markt « gegen das Prinzip »Staat «.

Die Beziehung zwischen Staat und Markt wird als Problem diskutiert, seit es Staaten gibt und Politik und Wirtschaft zu getrennten Sphären wurden. Zum Idealtypus des kapitalistischen Marktes gehört der Wettbewerb, und zwar Wettbewerb zwischen privaten Akteuren, die profitorientiert handeln. Ein rücksichtslos vom Eigeninteresse gelenktes Handeln kennzeichnete für Thomas Hobbes den menschlichen Urzustand, den er radikal negativ sah und der durch die gemeinsame, paktierte Unterordnung unter den Staat überwunden wird. Sein Leviathan spiegelt eine wertende Gegenüberstellung von Markt und Staat wider, die sich vom 17. bis ins 19. Jahrhundert verfolgen lässt. Während »Markt « dabei für die Dominanz von Einzelinteressen steht, steht "Staat « für Ordnung und Gemeinwohl. Diese wertende Gegenüberstellung kommt im 19. Jahrhundert noch in der Staats- und Gesellschaftslehre des Lorenz von Stein zum Ausdruck. Für Lorenz von Stein bedroht die egoistische Verfolgung individueller Interessen die soziale Gemeinschaft; der Staat, der das Wohl aller zu fördern sucht, unterwirft die Einzelinteressen kraft seiner Macht. Ein Markt schafft kein Wir: Der Staat repräsentiert das Wir.

Neben der wertenden Gegenüberstellung von Markt und Staat, in der Markt negativ und Staat als ordnende Macht positiv gewertet wird, stand lange Zeit eine zweite, umgekehrt wertende Gegenüberstellung, bei der Markt für Freiheit und Staat für Gewalt steht. Diese Gegenüberstellung ist uns aus der Ideologie des Neoliberalismus vertraut. Sie findet sich aber bereits im 16. Jahrhundert bei dem französischen Humanisten Jean Bodin: Der Staat gründet für Bodin auf Eroberung und Gewalt, wirtschaftliches Handeln dagegen beruht auf dem Prinzip der Kooperation - eine Auffassung, bei der die Erinnerung an mittelalterliche Zünfte mitschwingen mag. Albert Hirschman ${ }^{9}$ hat sehr gut nachgezeichnet, wie im Lauf des 16. und 17. Jahrhunderts aus wirtschaftlicher Tätigkeit, die der Aristokratie in der Feudalgesellschaft als ehr- und ruhmlos galt, und aus der Gier nach Geld und Besitz, die nach Augustinus eine der Kardinalsünden ist, eine »sanfte Kraft « wurde, die die anderen sündigen Leidenschaften zügelt, den Beziehungen unter Menschen Beständigkeit und Berechenbarkeit verleiht und so am Ende zu Frieden und Wohlstand führt. Typisch für diese Sicht der Dinge war der schottische Sozialphilosoph Adam Ferguson, der in der 2. Hälfte des 18. Jahrhunderts schrieb. Ferguson zählte den Selbsterhaltungstrieb, der nicht nur Individuen, sondern auch Gruppen eigen ist, zu den Grundursachen von Krieg und Konflikt; Gewerbe und Handel, die »Quellen des Reichtums «, basieren dagegen auf Frieden und dem »Fortschreiten bürgerlicher und gewerblicher Künste «. ${ }^{10}$ Die Annahme, dass Markt zu Frieden führt, ist Kernbestandteil liberalen Denkens. Auch der gemeinsame europäische Markt sollte nicht

8 Vgl. zum Beispiel Quaglia 2010; Quaglia 2012.

9 Hirschmann 1977.

10 Wobei dieser Fortschritt für Ferguson auf Arbeitsteilung beruht. Damit sind zwei Faktoren benannt, die bei vielen der frühen Sozialtheoretiker eine große Rolle spielten: Arbeitsteilung und Kampf der Interessen. 
nur wirtschaftliches Wachstum bringen, sondern eine Grundlage für Frieden in Europa sein. Dies ist in der Tat seine unbestrittene historische Leistung.

Markt und Staat waren nicht immer ein Gegensatzpaar. Die Metapher der unsichtbaren Hand von Adam Smith vermittelt den Gegensatz von Individualinteresse und Gemeinwohl ohne Rückgriff auf den ordnenden Staat, indem sie individuelle Interessenverfolgung automatisch zu kollektivem Wohlstand führen lässt. Das sah Marx anders, aber auch für ihn ist der Staat keine eigenständige Kraft, kein Gegenspieler des Marktes, sondern ein bloßes Epiphänomen - die Politik wird bei ihm zum verlängerten Arm des Kapitals, und der Gegenspieler ist das unterdrückte Proletariat.

Aus den unterschiedlichen ideengeschichtlichen Auffassungen der Natur von Markt und Staat und des Verhältnisses zwischen ihnen ergibt sich für beide eine grundsätzliche Ambivalenz. »Staat« steht einerseits für Gemeinwohl und Ordnung, andererseits für Gewalt und Unterdrückung, » Markt « steht für individuelle Freiheit, Frieden und Wohlstand ebenso wie für Egoismus, Konkurrenz und Ausbeutung. Eine gewisse Ambivalenz kennzeichnet auch die Wahrnehmung von Markt und Staat in der EU. Der gemeinsame europäische Markt produziert Wohlstand, erzeugt aber auch Probleme für einzelne Branchen. Der »Staat « - in Gestalt der politischen Institutionen der EU - wirkt ordnend, ist aber oft übermäßig restriktiv. Anders als bei Adam Smith erscheint die ordnende Hand der Politik in der EU nicht als überflüssig, und sie dient, anders als bei Karl Marx, nicht lediglich dem Kapital.

Ideengeschichtlich handelt es sich bei Markt und Staat um Prinzipien sozialer Ordnung. Man muss jedoch unterscheiden zwischen Markt und Staat als Ordnungsoder Gestaltungsprinzipien einerseits und als konkrete soziale Handlungszusammenhänge andererseits. Die Wirtschaft als konkreter sozialer Handlungszusammenhang ist in kaum einem Land rein als "Markt " organisiert, und reale politische Systeme sind selten strikt hierarchisch. Mehr noch: Markt und Staat, Wirtschaft und Politik sind nicht nur wechselseitig aufeinander angewiesen, sie konstituieren sich gegenseitig. Es gibt keine völlig politikfreien Märkte - und keine politischen Regime ohne ökonomische Basis. ${ }^{11}$ Dennoch sorgen die gegensätzlichen Ordnungsprinzipien Markt und Staat für eine ständige Spannung in den Verhandlungen der Mitgliedstaaten der EU bei der Suche nach gemeinsamen Entscheidungen.

Als Grund für die allfälligen Kooperationsprobleme in der EU werden in der Regel divergierende Interessen der Mitgliedstaaten als quasi unitarischen Akteuren genannt. ${ }^{12}$ Auf der letzten Jahreskonferenz der europäischen Politologenvereinigung ECPR etwa wurde festgestellt, dass Kooperation bei der Regulierung, der For-

11 Besonders nachdrücklich vertritt Jürgen Kocka (2013) die wechselseitige Beziehung zwischen Wirtschaft und Politik in seiner Geschichte des Kapitalismus.

12 Das ist, wie Martin Höpner (private Mitteilung) meint, durchaus bemerkenswert, denn im Prinzip wäre denkbar, dass sich unterschiedliche Strategien der nationalen Regierungen je nach der dominanten Parteizugehörigkeit entlang der Links-Rechts-Achse sortieren, was für die Bildung europäischer Parteien mit kohärenten Programmen förderlich wäre und die Art der Auseinandersetzungen in internationalen Gremien verändern würde. 
mulierung und Verabschiedung von für alle verbindlichen Regeln in der EU ständig durch strategische Überlegungen der Mitgliedsländer und durch ihr Beharren auf den je eigenen, nationalen Interessen unterlaufen würde. ${ }^{13}$ Kooperation setzt nicht notwendig Verhandlung voraus; Kooperation findet zum Beispiel auch im Fall der arbeitsteiligen Bewältigung einer Aufgabe statt, bei der jeder Partner seine speziellen Fähigkeiten einbringt. Divergieren jedoch die Interessen der Kooperationspartner bei der Verfolgung eines gemeinsamen Ziels, dann müssen sie miteinander verhandeln. Von den Repräsentanten der Mitgliedsländer, die in den verschiedenen europäischen Gremien miteinander über eine neue Direktive, eine praktische Maßnahme verhandeln, wird erwartet, dass sie das vertreten, was sie jeweils als ihre nationalen Interessen definieren. Das wiederum hängt mit dem Charakter der EU zusammen, die sich in ihren wesentlichen Zügen als institutionalisiertes Verhandlungssystem darstellt.

In der soziologischen Gesellschaftstheorie werden (im Unterschied zu der nur mit dem Gegensatzpaar Markt/Hierarchie operierenden Transaktionskostenökonomie) mindestens drei Typen sozialer Ordnungsbildung unterschieden, nämlich Ordnungsbildung durch hierarchische Anordnung, durch Verhandlung oder durch massenhafte individuelle Anpassung. Wird nicht von Interaktionsformen, sondern von Strukturmustern gesprochen, steht anstelle von Hierarchie Staat und anstelle von individueller Anpassung Markt. ${ }^{14}$ Verhandeln steht zwischen dem Hierarchieprinzip des Befehlens und dem Marktprinzip autonomen adaptiven Handelns und ist der Spannung zwischen diesen beiden Extremen ausgesetzt. ${ }^{15}$ Die EU ist durch zwischenstaatliche Verträge geschaffen worden: Sie ist weder ein bloßer Markt noch ein Staat. Zwar lassen sich innerhalb der EU auch Prozesse gegenseitiger Anpassung und hierarchischer Anordnung beobachten, ${ }^{16}$ aber was die Regulierung, die europäische Gesetzgebung angeht, ist die EU im Wesentlichen als Verhandlungssystem ausgebildet: ${ }^{17}$ Gemeinsame Entscheidungen kommen, selbst wenn am Ende nach dem Mehrheitsprinzip abgestimmt wird, typischerweise durch Verhandlung zwischen Vertretern der Mitgliedstaaten zustande. ${ }^{18}$ Am klarsten kommt das bei der Produktion europaweit verbindlicher Entscheidungen zum Ausdruck, bei der der

13 Vgl. Call for Papers der Sektion Regulatory Governance für diese Konferenz.

14 Mayntz, Scharpf 1995, S. 60-65.

15 Ähnlich sieht Albert Hirschman (1977) das Konzept des »Interesses « zwischen den gegensätzlichen Polen »Vernunft « und »Leidenschaft « stehen - und Elemente von beiden spannungsreich in ihm enthalten.

16 Scharpf 2010, S. 199-208.

17 Fritz Scharpf (2010, S. 201-207) unterscheidet zwischen »intergovernmental negotiation «, was für ihn die niedrigste Ebene von Institutionalisierung darstellt, und »joint decision «; tatsächlich findet im Prozess gemeinsamen Entscheidens ständig Verhandlung statt. Verhandlung und Verhandlungssysteme sind ein wichtiges Thema in der Politikwissenschaft; vgl. zum Beispiel Benz et al. 1992.

18 Vgl. hierzu auch die Sondernummer 5/2000 des Journal of European Public Policy, Negotiation and policy-making in the European Union. Zur politischen Bedeutung von Verhandlung, sprich »Verhandlungsdemokratie a allgemein vgl. auch Czada et al. 1993. 
Europäische Rat und der jeweilige Ministerrat die dominante Rolle spielen. ${ }^{19}$ Bei der Produktion verbindlicher Entscheidungen in Verhandlungssystemen verfolgen die Mitglieder einerseits eigene Interessen, als Mit-Glieder eines größeren Ganzen jedoch zugleich ein gemeinsames Ziel. Das gemeinsame Ziel setzt der Verfolgung von Partikularinteressen Grenzen. ${ }^{20}$ Gemeinsame Entscheidungen müssen eine Balance zwischen beiden finden.

Aber was sind die »nationalen "Interessen, die in der EU als partikulare Interessen gemeinsame Entscheidungen erschweren, wenn nicht gar verhindern? Auf der Suche nach einer Antwort auf diese Frage will ich einige Entscheidungen näher betrachten, die sich auf einen Kernbereich von Wirtschaft beziehen - die europäische Finanzindustrie und ihre Regulierung.

Der Übergang von einer Politik der Marktschaffung zu einer Politik der Marktregelung fand beim Finanzmarkt erst spät statt. Regulierung bedeutete hier noch gegen Ende des vorigen Jahrhunderts, als der Financial Services Action Plan (FSAP) verabschiedet wurde, im Wesentlichen die Schaffung eines gemeinsamen Marktes für Finanzdienstleistungen. Getragen war der FSAP vom Wunsch der Staaten nach höheren Erträgen durch einen integrierten Finanzmarkt, stieß bei ihnen aber zugleich wegen des drohenden Verlusts an nationalen Kompetenzen bei Regulierung und Aufsicht auf Widerstand. ${ }^{21}$ Mit der Finanzmarktkrise und ihren Folgen für europäische Banken und Staaten schwang das Pendel auch hier schließlich in Richtung Regulierung aus. Reguliert werden sollten jetzt auch die bislang nicht regulierten Hedgefonds. ${ }^{22} \mathrm{Im}$ Gegensatz zu Banken wurde Hedgefonds zunächst keine besondere Schuld am Entstehen der Finanzmarktkrise zugeschrieben. Selbst nach dem Beginn der Krise gab es in Europa noch Stimmen, die gegen ihre Regulierung sprachen. Nicht zuletzt Deutschland, das erst 2004 die rechtlichen Rahmenbedingungen für die Einrichtung von Hedgefonds geschaffen hatte, setzte sich dagegen schon länger erfolglos für eine Regulierung von Hedgefonds ein. Bis zum Ausbruch der Krise war die zentrale Streitfrage in der EU - der Primat von "Markt " oder "Staat «-, was Hedgefonds angeht, zugunsten des Marktprinzips beantwortet worden. Die Hedgefonds-Branche selbst war überrascht, als entgegen ihrer Einschätzung der Situation dann doch eine europäische Initiative zu ihrer Regulierung zustande kam.

Betrachtet man die Positionen der verschiedenen Länder bei den Verhandlungen über den Inhalt einer neuen Hedgefonds-Regulierung, dann wird deutlich, dass hier handfeste wirtschaftliche Interessen am Werk waren. Diese Interessen waren aber sehr unterschiedlich, was nicht zuletzt mit der verschiedenen Bedeutung von Hedgefonds in den einzelnen Ländern zusammenhängt. In der EU werden 76 Prozent der Hedgefonds-Industrie von London aus geleitet; registriert sind sie meist in Steuer-

19 Costello, Thomson 2013.

20 Diese Konstellation entspricht der spieltheoretischen Figur der »Battle of the Sexes «, wo bei unterschiedlichen individuellen Präferenzen zum Beispiel im Hinblick auf Reiseziele bei beiden Spielern die Präferenz dominiert, etwas gemeinsam zu unternehmen.

21 Quaglia 2010; Story, Walter 1997.

22 Zum Folgenden Woll 2012.

Leviathan, 42. Jg., 2/2014 
paradiesen außerhalb der EU. Deshalb war Großbritannien nicht nur für eine »sanfte " Regulierung, sondern auch darauf bedacht, diesen Fonds den Zugang zum europäischen Markt (in der Form eines »europäischen Passes «) zu erhalten. Nun hat die britische Finanzindustrie traditionell einen guten Draht zur britischen Politik, ${ }^{23}$ aber sie musste die Regierung gar nicht groß drängen, ihre Interessen zu vertreten. Großbritannien hat ein ureigenes politisches Interesse am Wohlergehen seiner Finanzindustrie. Natürlich erstreben alle Mitgliedstaaten der EU das Wohl ihrer eigenen Volkswirtschaft. Aber Wachstum beruht je nach Land auf verschiedenen Voraussetzungen, die von der Struktur der eigenen Wirtschaft abhängen: Es gibt kein anderes europäisches Land, in dem die Finanzindustrie als Steuerquelle und Arbeitgeber eine derart wichtige Rolle spielt wie in Großbritannien.

Etwas anders lag der Fall in Frankreich. In Frankreich gab es neben einigen großen Hedgefonds zahlreiche kleinere, regulierte Fonds (sogenannte UCITS-Fonds). Dieser Sektor fürchtete die Konkurrenz der großen, nichtregulierten Hedgefonds und unterstützte deshalb den Plan ihrer Regulierung; alle französischen Investmentfonds wollten außerdem auswärtigen Hedgefonds den Zugang zum europäischen Markt, das heißt den »europäischen Pass ", verweigern. Die bereits regulierten UCITSFonds hatten Erfahrung mit dem Regulierungsverfahren in der EU; sie schlossen sich zu einer Lobby zusammen und artikulierten ihr Interesse der damaligen Finanzministerin Lagarde und dem Präsidenten Sarkozy gegenüber, die diese Position dann auch in den Verhandlungen vertraten. Zwar kann man auch hier nicht von »capture « sprechen, der Vereinnahmung von Politik durch ein Partikularinteresse, aber die organisierte Interessenvertretung spielte offenbar eine wichtige Rolle bei der Definition des französischen Interesses an einer künftigen Hedgefonds-Regulierung.

Grundsätzlich gibt es zwei Kanäle für den Einfluss ökonomischer Interessen auf die Verhandlungsposition von Regierungen bei Regulierungsentscheidungen: den Weg über Verbände und Lobbies und den Weg über die unvermittelte politische Wahrnehmung der - erwünschten ebenso wie unerwünschten - Folgen des faktischen Verhaltens ökonomischer Akteure. ${ }^{24}$ Ein Beispiel für den zweiten Fall bietet auch die Position von Deutschland in der Regulierung von Hedgefonds, nur dass es hier anders als in Großbritannien nicht um ein politisches Interesse am Wohlergehen der Finanzindustrie ging. Vielmehr war in Deutschland schon länger das Verhalten einer speziellen Kategorie von Fonds, den als »Heuschrecken « verteufelten Beteiligungsgesellschaften, von namhaften Politikern öffentlich kritisiert worden. Eine Hedgefonds-Regulierung bot die Möglichkeit, etwas zur Lösung dieses von der Politik erkannten Problems zu tun. Deshalb war es das Ziel Deutschlands bei den Verhandlungen, durch strikte Regulierung diese Fonds an dem sogenannten asset stripping zu hindern, dem schnellen Verkauf von Vermögensbestandteilen übernommener Unternehmen, durch den Schulden zurückbezahlt werden, die für die

23 Vgl. Johal et al. 2012.

24 Die Beeinflussung von Politikern durch Bestechung statt durch Überzeugung mag in der Politik der USA aufgrund der Bedeutung von Wahlkampfspenden häufiger anzutreffen sein als beispielsweise in Großbritannien oder Deutschland; dabei mögen auch kulturelle Unterschiede bis hin zur Wahrnehmung des Staates eine Rolle spielen. 
Übernahme bei den Banken gemacht wurden. Am Ende der Verhandlungen über die europäische Hedgefonds-Regulierung stand - natürlich - ein Kompromiss: Die am 1. Juli 2011 in Kraft getretene europäische Direktive ist strikter, als Großbritannien es wünschte, und nicht ganz so strikt, wie Deutschland es gern gehabt hätte; es wird einen »europäischen Pass « für auswärtige (Nicht-EU-)Hedgefonds geben, der ihnen den Zugang zum europäischen Markt öffnet - aber erst nach einer mehrjährigen Übergangszeit und nicht bedingungslos.

Während fiskalische und volkswirtschaftliche Staatsinteressen bei der Hedgefonds-Regulierung eine zentrale Rolle spielten, ging es bei der europäischen Regulierung von Ratingagenturen zunächst vor allem um Regelungskompetenzen, also um Macht. Zur Zeit der Finanzmarktkrise dominierten weltweit die drei großen amerikanischen Agenturen, es gab also keine direkt betroffene europäische Branche. Ratingagenturen wurde zwar - anders als Hedgefonds - von Anfang an Mitschuld am Entstehen der Finanzmarktkrise gegeben. Es gab jedoch, ebenfalls im Unterschied zu Hedgefonds, bereits einen von der Internationalen Organisation von Wertpapierbehörden IOSCO entworfenen Verhaltenskodex, den viele, sofern er denn eingehalten würde, auch in Europa zunächst für nur geringfügig verbesserungsbedürftig hielten. ${ }^{25}$ Die Debatte konzentrierte sich deshalb auf die Frage, wie die Einhaltung der Verhaltensregeln kontrolliert wird, durch die einzelnen Mitgliedstaaten oder durch eine neu zu schaffende europäische Aufsichtsinstanz, an die sie ihre Kompetenzen abgeben würden. Die Entscheidung fiel in diesem Fall ohne größere Konflikte zugunsten der europäischen Lösung. Dagegen wurde bei der Schaffung der Europäischen Bankenunion höchst konfliktreich um Kompetenzen gestritten, etwa als es darum ging, ob es eine harte, gesamteuropäische Regel über den Umfang geben soll, in dem Aktionäre, Gläubiger und Einleger im Fall der Abwicklung einer maroden Bank für Verluste haften, oder ob die einzelnen Länder hier noch einen Entscheidungsspielraum haben. Wie nicht nur im Spiegel zu lesen war, stritten nicht zufällig gerade die EU-Staaten mit den größten Banken am heftigsten um einen eigenen Entscheidungsspielraum. ${ }^{26}$ Um nationale Kompetenzen wird gestritten, wenn der Regelungsgegenstand, um den es geht, im eigenen Land praktische Bedeutung hat.

Was also sind, grob und vorsichtig formuliert, die "nationalen « Interessen, deren Vertretung die Kooperation beim Fällen gemeinsamer Entscheidungen erschwert? Die Ziele, die die Repräsentanten verschiedener Länder bei den Verhandlungen über gemeinsame Entscheidungen verfolgen, sind ebenso wirtschaftlicher wie politischer Natur - wobei, da das politische Gewicht eines Landes nicht zuletzt vom Gewicht seiner Wirtschaft abhängt, die Förderung der eigenen Wirtschaft dem politischen Ziel dienen kann, den »komparativen Vorteil « eines Landes im internationalen Wettbewerb zu wahren. ${ }^{27}$ Inwieweit es sich allerdings bei den in politischen Verhandlungen vertretenen wirtschaftlichen Interessen um ökonomische »Staatsinter-

25 Vgl. De Haan, Amtenbrink 2011.

26 Der Spiegel 2013.

27 Fioretos 2001.

Leviathan, 42. Jg., 2/2014 
essen " und nicht um »capture ", also um unter dem Druck von Verbänden und Lobbys von der Politik vertretene wirtschaftliche Einzelinteressen handelt, mag im Einzelfall schwer zu entscheiden sein. Denn natürlich müssen ökonomische Staatsinteressen definiert werden. »Capture « liegt nur vor, wenn es Partikularinteressen gelingt, den auf ein allgemeines Wohl zielenden politischen Entscheidungsprozess im eigenen Interesse umzubiegen. ${ }^{28}$ Politiker mögen jedoch wie im Fall der sich nachträglich als krisenträchtig erweisenden Deregulierung der Finanzindustrie von sich aus der Überzeugung sein, im allgemeinen Wohl zu handeln, wenn sie ein bestimmtes wirtschaftliches Interesse fördern; James Kwak ${ }^{29}$ spricht hier von »cultural capture«. Wenn Wachstum politisch Priorität hat, haben wirtschaftliche Interessen ein besonderes Gewicht. Aber man sollte die Bedeutung des seit Gründung der EU immer virulenten Autonomiebestrebens insbesondere größerer Mitgliedsländer als Motiv bei der Auseinandersetzung um Regulierungsentscheidungen nicht unterschätzen. Es ist durchaus möglich, dass gerade die immer stärker werdenden Eingriffe in nationale Kompetenzbereiche bei den Mitgliedstaaten reaktiv zu einem Beharren auf nationaler Autonomie führen; Politikwissenschaftler sehen hier sogar Anzeichen für einen Wandel in der Wahrnehmung dessen, was »Macht « für einen Nationalstaat heißt, nämlich nicht Einfluss auf andere zu haben, sondern Autonomie zu bewahren. ${ }^{30}$

Bisher habe ich von den Interessen gesprochen, die verschiedene Länder in Verhandlungen vertreten. Es sei jedoch wenigstens kurz daran erinnert, dass die Struktur, in der solche Verhandlungen stattfinden, und die Verfahrensregeln, denen sie unterworfen sind, die europäischen Kooperationsprobleme tendenziell noch erhöhen. ${ }^{31}$ Bei der legislativen Regulierung, bei der nach der sogenannten »Gemeinschaftsmethode « entschieden wird, wird die gleiche Sache von verschiedenen Akteuren in verschiedenen Gremien verhandelt - in der Kommission durch Beamte, im Europäischen Rat beziehungsweise dem zuständigen Ministerrat durch Regierungsvertreter und im Europäischen Parlament durch gewählte Abgeordnete. ${ }^{32}$ Dabei sind Kommission und Parlament keine neutralen Foren, in denen nationale Interessen wie im zuständigen Ministerrat direkt aufeinandertreffen. Kommission und Parlament entwickeln vielmehr eigene Interpretationen des gemeinsamen Ziels und der infrage stehenden nationalen Interessen, wobei nationale Interessen in der Kommission vor allem in dem stark entwickelten Ausschusswesen zu Wort kommen, ${ }^{33}$ während sich bei den Parlamentariern nationale mit parteipolitischen Präferenzen mischen. Außerdem verfolgen Kommission wie Parlament nicht selten eigene Machtinteressen. Sowohl Konflikte um nationale oder europäische Regelungskompetenz als auch Konflikte um die Priorität von Markt (Marktschaffung) oder Staat

28 Mattli, Woods 2009.

29 Kwak 2014.

30 Cohen 2006, zitiert in Helleiner, Pagliari 2011.

31 Vgl. hierzu auch Joerges 2012, S. 371-373, S. 376-381.

32 Vgl. unter anderem Fabbrini 2013.

33 Comitology; vgl. Neyer 2000. 
(politische Regelung) werden im Institutionensystem der EU somit vielfach gebrochen.

Aus all dem wird deutlich, dass es auf die im Titel gestellte Frage, ob die Ursache europäischer Kooperationsprobleme eher mit »Markt « oder mit »Staat " zusammenhängt, keine einfache Antwort gibt. Der Gegensatz von Markt und Staat manifestiert sich in der EU auf doppelte Weise - in den Auseinandersetzungen um die fallweise Priorität von Schaffung oder Regelung eines Marktes, vor allem aber als Konflikt zwischen Einzel- und Gemeininteressen, wenn es um europäische Entscheidungen geht. In Verhandlungssystemen sind bei jeder gemeinsamen Entscheidung Konflikte mit partikularen Interessen vorprogrammiert. Und das gilt nicht nur für Regulierungsentscheidungen wie in meinen konkreten Beispielen, sondern nach wie vor genauso für Entscheidungen zur Schaffung, Erweiterung oder Vertiefung von Märkten. In einem nicht nur, aber eben auch als Verhandlungssystem institutionalisierten politischen Gebilde wie der EU ist die Vertretung nationaler Einzelinteressen legitim; Gustavsson ${ }^{34}$ spricht deshalb auch vom »legitimen Protektionismus " der Mitgliedstaaten. Ein legitimer ökonomischer Nationalismus muss jedoch dort eine Grenze finden, wo er einem europäischen Wirtschaftspatriotismus zuwiderläuft ${ }^{35}$ und die Kooperation in der EU untergräbt.

Die notwendige Balance zwischen staatlichen Einzelinteressen und gemeinsamen europäischen Interessen zu finden wird schwieriger, wenn in Krisenzeiten die ökonomische "Integrationsdividende", der Nutzen, den ihre Mitgliedschaft in der Union den einzelnen Mitgliedstaaten bringt, sinkt oder sich gar in messbare Kosten verkehrt und anstelle des für Kooperation charakteristischen Gebens und Nehmens ein entschädigungsloses Geben, also Solidarität treten soll. Wenn das gemeinsame Ziel verblasst, verkommt Kooperation zum bloßen Tauschhandel zwischen divergierenden nationalen Interessen. Nicht zufällig ist in letzter Zeit häufiger von einem möglichen Zerfall der EU die Rede. ${ }^{36}$ Könnte es sein, dass die EU im Begriff ist, ihre Basis, ein wirklich von allen Mitgliedern verfolgtes gemeinsames Ziel zu verlieren? In der schon einmal zitierten Schrift des Zentrums für globale Kooperationsforschung heißt es, dass »[...] cooperation works very well within a social group if this group constitutes itself against another group « - Kooperation funktioniert dann in einer sozialen Gruppe gut, wenn diese Gruppe sich gegen eine andere Gruppe richtet. ${ }^{37}$ Ein gemeinsamer Feind hat, wie die Geschichte lehrt, etwas ungemein Verbindendes; einen Feind, eine Bedrohung von außen auszumachen ist eine beliebte politische Strategie, wenn der innere Zusammenhalt bedroht ist. Kein verantwortungsbewusster Europapolitiker wird eine solche Strategie empfehlen. Aber man mag Walter Laqueur ${ }^{38}$ zustimmen, wenn er beklagt, dass der politische Akteur EU

\section{Gustavsson 2014.}

35 Clift, Woll 2013.

36 So fand zum Beispiel am 28. Oktober 2013 im Institut für Europäische Integrationsforschung in Wien eine Tagung über »European Integration - and Disintegration? « statt.

37 Weinlich 2013, S. 24.

38 Laqueur 2013.

Leviathan, 42. Jg., 2/2014 
sich zu sehr mit sich selbst beschäftigt und eine weltpolitische Rolle nicht spielen will. Ist die Politik der EU zu selbstbezogen - und zugleich zu wenig politisch? Vielleicht geht es gar nicht in erster Linie um Markt oder Staat im Inneren der EU, sondern um die Position der EU in einer von Spannungen geprägten und Kämpfen zerrissenen Welt - einer Welt, die etwas anderes ist als nur ein globaler Markt.

\section{Literatur}

Benz, Arthur; Scharpf, Fritz W.; Zintl, Reinhard 1992. Horizontale Politikverflechtung. Zur Theorie von Verhandlungssystemen. Frankfurt a. M., New York: Campus.

Centre for Global Cooperation Research 2013. Annual Report 1. Duisburg: Käte Hamburger Kolleg.

Clift, Ben; Woll, Cornelia. Hrsg. 2013. Economic patriotism in open economies. London: Routledge.

Costello, Rory; Thomson, Robert 2013. »The distribution of power among EU institutions: who wins under co-decision and why? «, in Journal of European Public Policy 20, 7, S. 1025-1039.

Czada, Roland; Schmidt, Manfred; Lehmbruch, Gerhard. Hrsg. 1993. Verhandlungsdemokratie, Interessenvermittlung, Regierbarkeit. Opladen: Westdeutscher Verlag.

De Haan, Jacob; Amtenbrink, Fabian 2011. Credit rating agencies. DNB Working Paper Nr. 278. Amsterdam: De Nederlandsche Bank.

Der Spiegel 2013. »Aufmarsch der Blockierer «, in Der Spiegel 30, S. 58-60.

Eberlein, Burkard; Grande, Edgar 2003. "Die Europäische Union als Regulierungsstaat «, in Europäische Union, hrsg. v. Jachtenfuchs, Markus; Kohler-Koch, Beate, S. 417-448. Opladen: Westdeutscher Verlag.

Fabbrini, Sergio 2013. "Intergovernmentalism and its limits ", in Comparative Political Studies 46, 9, S. 1003-1029.

Fioretos, Orfeo 2001. »The domestic sources of multilateral preference: varieties of capitalism in the European Community «, in Varieties of capitalism. The institutional foundations of comparative advantage, hrsg. v. Hall, Peter A.; Soskice, David, S. 213-244. Oxford: Oxford University Press.

Gustavsson, Sverker 2014. "Democratic reformism presupposes legitimate protectionism «, in Democratic politics in a European Union under stress", hrsg. v. Cramme, Olaf; Hobolt, Sarah B. Oxford: Oxford University Press (im Erscheinen).

Helleiner, Eric; Pagliari, Stefano 2011. »The end of an era in international financial regulation? A postcrisis research agenda ", in International Organization 65, S. 169-200.

Hirschman, Albert O. 1977. The passions and the interests. Political arguments for capitalism before its triumph. Princeton: Princeton University Press.

Joerges, Christian 2012. "Europas Wirtschaftsverfassung in der Krise«, in Der Staat 51, S. 357-386.

Johal, Subhdev; Moran, Michael; Williams, Karel 2012. "Post-crisis financial regulation in Britain ", in Crisis and control. Institutional change in financial market regulation, hrsg. v. Mayntz, Renate, S. 67-95. Frankfurt a. M.: Campus.

Kocka, Jürgen 2013. Geschichte des Kapitalismus. München: C. H. Beck.

Kratochvil, Petr. Hrsg. 2013. The EU as a political actor. Baden-Baden: Nomos.

Kwak, James 2014. "Cultural capture and the financial crisis ", in Preventing regulatory capture. Special interest influence and how to limit it, hrsg. v. Carpenter, Daniel; Moss, David A., S. 71-98. Cambridge: Cambridge University Press.

Laqueur, Walter 2013. Europa nach dem Fall. München: Herbig.

Lepsius, Rainer 2013. "In welchen Krisen befindet sich die Europäische Union? «, in Zeitschrift für Politik 60, 2, S. 182-193.

Mattli, Walter; Woods, Ngaire 2009. »In whose benefit? Explaining regulatory change in global politics «, in The politics of global regulation, hrsg. v. Mattli, Walter; Woods, Ngaire, S. 1-43. Princeton: Princeton University Press. 
Mayntz, Renate; Scharpf, Fritz W. 1995. „Der Ansatz des akteurzentrierten Institutionalismus «, in Gesellschaftliche Selbstregelung und politische Steuerung, hrsg. v. Mayntz, Renate; Scharpf, Fritz W., S. 39-72. Frankfurt a. M.: Campus.

Mayntz, Renate. Hrsg. 2012. Crisis and control. Institutional change in financial market regulation. Frankfurt a. M.: Campus:

Mayntz, Renate 2013. Financial market regulation in the shadow of the sovereign debt crisis. MPIfG Discussion Paper 13/11. Köln: Max-Planck-Institut für Gesellschaftsforschung.

Neyer, Jürgen 2000. "Justifying comitology: the promise of deliberation ", in European integration after Amsterdam, hrsg. v. Neunreither, Karlheinz; Wiener, Antje, S. 118-128. Oxford: Oxford University Press.

Quaglia, Lucia 2010. Governing financial services in the European Union: banking, securities, and post-trading. London: Routledge.

Quaglia, Lucia 2012, »The regulatory response of the European Union to the global financial crisis", in Crisis and control. Institutional change in financial market regulation, hrsg. v. Mayntz, Renate, S. 171-195. Frankfurt a. M.: Campus.

Scharpf, Fritz W. 1999. Governing Europe: effective and democratic? Oxford: Oxford University Press.

Scharpf, Fritz W. 2010. Community and autonomy. Institutions, policies and legitimacy in multilevel Europe. Frankfurt a. M.: Campus.

Schmidt, Susanne K. 2007. "Mutual recognition as a new mode of governance ", in Journal of European Public Policy 14, 5, S. 667-681.

Story, Jonathan; Walter, Ingo 1997. Political economy of financial integration in Europe - the battle of the systems. Manchester: Manchester University Press.

Weinlich, Silke 2013. "Global we? New insights about the foundations of global cooperation «, in Global cooperation - Re:Search 2012/13. Annual Report 1, S. 24-25. Duisburg: Käte Hamburger Kolleg/Centre for Global Cooperation Research.

Woll, Cornelia 2012. »The defense of economic interests in the European Union: the case of hedge fund regulation ", in Crisis and control. Institutional change in financial market regulation, hrsg. v. Mayntz, Renate, S. 197-211. Frankfurt a. M.: Campus. 
Zusammenfassung: Auf dem Hintergrund ideengeschichtlicher Überlegungen zum Verhältnis von Markt und Staat wird nach den Ursachen der beständigen Kooperationsprobleme gefragt, mit denen es die Europäische Union zu tun hat, wenn für alle Mitgliedstaaten verbindliche Entscheidungen getroffen werden sollen. Die Tatsache, dass ein politisch gewollter gemeinsamer Markt die Basis der Europäischen Union ist, begründet ein grundsätzliches Spannungsverhältnis der Ordnungsprinzipien Markt und Staat. Am Beispiel von Entscheidungen zur Regulierung der Finanzmärkte wird gezeigt, dass politische ebenso wie ökonomische Interessen der Mitgliedstaaten hinter den dabei aufgetretenen Kooperationsproblemen stehen. Wirtschaftliche und politische Interessen stehen jedoch nicht in Gegensatz zueinander, sondern verschmelzen zu ökonomischen Staatsinteressen. Die Kooperationsprobleme in der Europäischen Union sind kein vermeidbarer Mangel, sondern hängen mit ihrer Struktur und Verfassung zusammen.

Stichworte: Kooperation, Verhandlungssysteme, Europäische Union, Finanzmarktregulierung

\section{State or market? Problems of cooperation in the EU}

Summary: A brief recapitulation of the century old discussion concerning the relation between »state « and » market « serves as background for the analysis of cooperation problems faced by the European Union. The fact that the EU as a political entity is built upon a common market creates a fundamental tension between economic and political principles in joint policy decision making. Using examples from financial market regulation, this article shows that the observed difficulties in reaching joint policy decisions have their roots both in political and economic interests which, instead of conflicting with each other, merge into economic state interests. The cooperation problems of the European Union follow unavoidably from its very constitution.

Keywords: cooperation, negotiation systems, European Union, financial market regulation

\section{Autorin}

Prof. Dr. Renate Mayntz

Max-Planck-Institut für Gesellschaftsforschung

Paulstr. 3

50676 Köln 